\title{
3D APT and NOE CEST-MRI of healthy volunteers and patients with non-enhancing glioma at $3 \mathrm{~T}$
}

\author{
Yulun Wu $\mathrm{W}^{1,2}$ (D) Tobias C. Wood $^{3} \cdot$ Fatemeh Arzanforoosh ${ }^{1,2}$. Juan A. Hernandez-Tamames ${ }^{1} \cdot$ Gareth J. Barker $^{3}$. \\ Marion Smits ${ }^{1,2}$. Esther A. H. Warnert ${ }^{1,2}$
}

Received: 30 June 2021 / Revised: 17 December 2021 / Accepted: 23 December 2021 / Published online: 7 January 2022

(c) The Author(s) 2021, corrected publication 2022

\begin{abstract}
Objective Clinical application of chemical exchange saturation transfer (CEST) can be performed with investigation of amide proton transfer (APT) and nuclear Overhauser enhancement (NOE) effects. Here, we investigated APT- and NOE-weighted imaging based on advanced CEST metrics to map tumor heterogeneity of non-enhancing glioma at $3 \mathrm{~T}$.

Materials and methods APT- and NOE-weighted maps based on Lorentzian difference (LD) and inverse magnetization transfer ratio $\left(\mathrm{MTR}_{\mathrm{REX}}\right)$ were acquired with a $3 \mathrm{D}$ snapshot CEST acquisition at $3 \mathrm{~T}$. Saturation power was investigated first by varying $\mathrm{B}_{1}(0.5-2 \mu \mathrm{T})$ in 5 healthy volunteers then by applying $B_{1}$ of 0.5 and $1.5 \mu \mathrm{T}$ in 10 patients with non-enhancing glioma. Tissue contrast (TC) and contrast-to-noise ratios (CNR) were calculated between glioma and normal appearing white matter (NAWM) and grey matter, in APT- and NOE-weighted images. Volume percentages of the tumor showing hypo/ hyperintensity $\left(\mathrm{VP}_{\text {hypo/hyper,CEST }}\right)$ in APT/NOE-weighted images were calculated for each patient.

Results LD APT resulting from using a $B_{1}$ of $1.5 \mu \mathrm{T}$ was found to provide significant positive $\mathrm{TC}_{\text {tumor,NAWM }}$ and MTR $\mathrm{REX}$ $\operatorname{NOE}\left(B_{1}\right.$ of $\left.1.5 \mu \mathrm{T}\right)$ provided significant negative $\mathrm{TC}_{\text {tumor,NAWM }}$ in tissue differentiation. $\mathrm{MTR}_{\mathrm{REX}}$-based NOE imaging under $1.5 \mu \mathrm{T}$ provided significantly larger $\mathrm{VP}_{\text {hypo,CEST }}$ than MTR $\mathrm{REX}_{\mathrm{R}}$ APT under $1.5 \mu \mathrm{T}$.

Conclusion This work showed that with a rapid CEST acquisition using a $B_{1}$ saturation power of $1.5 \mu \mathrm{T}$ and covering the

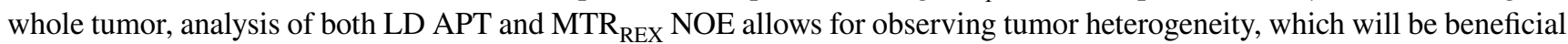
in future studies using CEST-MRI to improve imaging diagnostics for non-enhancing glioma.
\end{abstract}

Keywords CEST $\cdot$ APT $\cdot \mathrm{NOE} \cdot$ Non-enhancing glioma

\section{Introduction}

Chemical exchange saturation transfer (CEST) imaging is a novel MRI technique with great potential for glioma diagnostics. CEST is sensitive to a reduction of bulk water signal induced by saturation transfer from exchangeable

Yulun $\mathrm{Wu}$

y.wu@erasmusmc.nl

$\square$ Esther A. H. Warnert

e.warnert@erasmusmc.nl

1 Department of Radiology and Nuclear Medicine, Erasmus MC, Dr. Molewaterplein 40, 3015 GD Rotterdam, The Netherlands

2 Brain Tumor Centre, Erasmus MC Cancer Institute, Rotterdam, The Netherlands

3 Centre for Neuroimaging Science, King's College London, London, UK protons in a number of compounds, via which these compounds can be detected [1]. Some of these biological compounds are increased in tumor regions and can therefore potentially be used as biomarkers for tumors. One popular chosen biomarker is amide proton transfer (APT) imaging, which focuses on the amide protons of endogenous mobile proteins and peptides that resonate at $3.5 \mathrm{ppm}$ [2]. Previous work has shown that APT signal is closely related to increased cell density, proliferation [3-5], and concentrations of intracellular proteins in gliosarcoma [6, 7]. Additionally, APT-weighted CEST has already been shown to be of value for clinical diagnostics in glioma, including response assessment to treatment [8-10], predicting IDH mutation status for diagnosis [5, 11], and predicting overall survival and progression-free survival [12]. However, the majority of patients studied in the current body of literature is diagnosed with high grade, enhancing gliomas (i.e., glioblastoma) [9, 13-15]. Limited studies investigate 
non-enhancing glioma explicitly. However, non-enhancing glioma can become quite large with molecular intratumoral heterogeneity [16] and we previously illustrated that APTweighted CEST may play a role in imaging diagnostics in these type of tumors [17]. This is of particular importance in light of the latest World Health Organization classification for brain tumors [18], where three distinct classes of nonenhancing glioma are identified based on the identification of molecular parameters (mainly IDH mutation and $1 \mathrm{p} / 19 \mathrm{q}$ co-deletion). These classes significantly differ in terms of prognosis and optimal treatment regime, stressing the need for optimal imaging diagnostics where APT-weighted CEST can play an important role.

To evaluate APT-weighted CEST in glioma diagnostics, early studies proposed to apply magnetization transfer ratio asymmetry (MTR $\mathrm{Masym}_{\text {m }}$ ) to perform APT-weighted imaging $[4,19-21]$. MTR $_{\text {asym }}$ is therefore a commonly used metric to provide CEST-weighted images by quantifying the asymmetry of Z-spectra while compensating for direct water saturation (DS) and magnetization transfer (MT) effects [1, 2, 4, $19,20,22,23]$. However, MT is a dominant contributor to the CEST signal when using high saturation power [24] and research is increasingly indicating that MT is not symmetric [25-27], which results in MTR $_{\text {asym }}$ not fully correcting for MT. In addition, MTR $_{\text {asym }}$ cannot evaluate saturation pools on opposite sides of the main resonance frequency individually, i.e., the effect of APT (3.5 ppm) and nuclear Overhauser enhancement (NOE, at $-3.5 \mathrm{ppm}$ ) are both reflected in $\mathrm{MTR}_{\text {asym }}$ at $3.5 \mathrm{ppm}$. Advanced metrics were proposed for separation of the APT and NOE signal, via multi-pool Lorentzian fitting [28] and the isolation of individual CEST effects by the Lorentzian difference (LD) [17, 28-33]. In addition, relaxation-compensated inverse magnetization transfer ratio $\left(\operatorname{MTR}_{\operatorname{Rex}}[34,35]\right)$ combined with Lorentzian fitting was proposed in glioma imaging at clinical field strength (3 T) to account for spillover effects that cannot be compensated by LD analysis [13, 28, 30, 36-38].

The NOE effect arises from through-space inter- and intramolecular dipole-dipole magnetization transfer between the water protons and aliphatic and olefinic components of mobile proteins, peptides, metabolites, and lipids, which is different than conventional MT contrast [1]. NOE imaging has also shown potential to serve as a novel imaging biomarker for glioma grading [39], predicting early progression after treatment $[8,9]$, and mapping tumor heterogeneity [40]. Moreover, Paech et al. showed that NOE-weighted CEST contrast also correlates with histopathological assessments of cell density in glioblastoma [41].

Acquisition of CEST metrics via a Lorentzian fitting approach requires densely sampled frequency offsets across a wide range, which inherently increases scan time and hampers application in clinical settings. Recently, a snapshot 3D readout has been developed to obtain CEST contrast for an image volume covering several slices with an acquisition time of $7 \mathrm{~s}$ per irradiation frequency offset [42, 43]. This efficient acquisition allows dense sampling of Z-spectra with a wide range within clinically feasible scan time. When considering clinical feasibility of CEST in non-enhancing glioma imaging, it is important to understand the application of LD-based APT and NOE studies under an appropriate saturation module at $3 \mathrm{~T}$. Our initial work showed that APTweighted signal isolated from Lorentzian fitting of DS has the ability to map tumor heterogeneity by identifying areas of hyperintensity within non-enhancing glioma, even with a suboptimal saturation and acquisition scheme [17]. Building on this work, we now use 3D snapshot CEST [42, 43] and investigate APT and NOE imaging in non-enhancing glioma based on Lorentzian fitting of DS and MT. This was done first in healthy volunteers to fine tune the saturation module of the CEST acquisition scheme. Subsequently, we applied two different saturation powers $\left(B_{1}\right)$ and applied LD and MTR $_{\text {REX }}$ analysis in 10 patients diagnosed with non-enhancing gliomas to investigate and compare the ability of APTand NOE-weighted CEST-MRI to map tumor heterogeneity.

\section{Materials and methods}

Our studies were conducted in compliance with the Declaration of Helsinki and under approval of the institutional ethics committee of the Erasmus MC (Rotterdam, NL), which is 1 out of 18 accredited medical research ethics committees in the Netherlands. For the healthy volunteer study, we recruited five subjects (male/female $=1 / 4$, mean age: 24.9 years). For the patient study, ten subjects with nonenhancing glioma were recruited. Patients were recruited as part of the Imaging Genomics study [44] and were scanned at maximum 2 days before surgical resection, where biopsies were taken for tumor stratification. Patient characteristics can be seen in Table 1 .

\section{Image acquisition}

All measurements were performed on a 3 Tesla scanner (Discovery750, General Electric, Chicago, USA) with a 32-channel head coil. A 3D snapshot CEST sequence [43] was used with the following acquisition parameters: $\mathrm{TR}=7 \mathrm{~ms}$, $\mathrm{TE}=3.2 \mathrm{~ms}$, field of view $=220 \times 180 \times 42 \mathrm{~mm}^{3}$ and matrix size $128 \times 104 \times 14$ for a resolution of $1.7 \times 1.7 \times 3 \mathrm{~mm}^{3}$, acceleration factor of 4 , and flip angle 6 degrees. Pulse-train saturation consisted of a train of 80 Gaussian-shaped radiofrequency (RF) pulses, each pulse with pulse time $20 \mathrm{~ms}$ and interpulse delay $20 \mathrm{~ms}$ ( $50 \%$ duty cycle), resulting in $3.2 \mathrm{~s}$ total saturation time.

Z-spectra were obtained with 53 frequency offsets: at $\pm 100 \mathrm{ppm}$, from \pm 50 to $\pm 20 \mathrm{ppm}$ in steps of $10 \mathrm{ppm}$, 
Table 1 Information of patients included in our study

\begin{tabular}{lllllll}
\hline Subject & Age & M/F & Grade & Type & $\begin{array}{l}\text { 1p/19q co- } \\
\text { deletion }\end{array}$ & IDH mutation \\
\hline 1 & 32 & M & 2 & Oligodendroglioma & True & True \\
2 & 28 & F & 2 & Astrocytoma & False & True \\
3 & 31 & M & 2 & Astrocytoma & False & True \\
4 & 24 & M & 3 & Oligodendroglioma & True & True \\
5 & 35 & M & 2 & Astrocytoma & False & True \\
6 & 37 & M & 2 & Astrocytoma & False & True \\
7 & 30 & F & 2 & Astrocytoma & False & True \\
8 & 52 & M & 4 & Glioblastoma & False & False \\
9 & 46 & F & 2 & Oligodendroglioma & True & True \\
10 & 54 & M & 3 & Oligodendroglioma & True & True \\
\hline
\end{tabular}

from \pm 10 to $\pm 5 \mathrm{ppm}$ in steps of $1 \mathrm{ppm}$, from \pm 4 to \pm 1 in steps of $0.5 \mathrm{ppm}$, and from \pm 0.5 to $0 \mathrm{ppm}$ in steps of $0.25 \mathrm{ppm}$. In addition, two images were obtained with saturation pulses at $-300 \mathrm{ppm}$ (i.e., at a frequency at which any saturation effects are expected to be negligible). The first was discarded as the signal had not reached equilibrium. The second image was used to normalize the Z-spectrum. The CEST scan took $4 \mathrm{~min}$ and $40 \mathrm{~s}$.

To investigate appropriate $B_{1}$ saturation power of the CEST sequence, we applied varying $B_{1}$ saturation power $(0.5,0.75,1,1.5,2 \mu \mathrm{T})$ in the scan of each healthy volunteer. Additionally, we investigated $\mathrm{B}_{1}$ saturation duration and acquired two more CEST acquisitions using 40 or $60 \mathrm{RF}$ pulses under the $B_{1}$ of $2 \mu \mathrm{T}$. In patients diagnosed with glioma, we applied two $B_{1}$ saturation powers $(0.5,1.5 \mu \mathrm{T})$ and $80 \mathrm{RF}$ pulses to investigate tumor imaging and tumor-white matter separation dependent on $B_{1}$. Note that the saturation powers used are stated as the root mean square $B_{1}$ (RMS $B_{1}$ ) across the saturation train.

\section{Data analysis}

Motion correction of the CEST image series was done by linear registration of each image within a series to the $6 \mathrm{ppm}$ image (mcflirt [45], within the free online software FMRIB Software Library (FSL) v5.0.9 [46]). After motion correction, the CEST image at 6 ppm was linearly registered to the $T_{1}$-weighted post-contrast image, resulting in transformation matrices from CEST to $T_{1}$ post-contrast space. These matrices were inverted and used to transform the regions of interest (ROI) based on structural images into the CEST space. All alignments were linear registrations performed with FLIRT [45] within FSL.

In-house written scripts in Matlab (Version R2015a, the Mathworks, Inc., Natick, MA) were used to perform voxelwise analysis for the CEST image series. Z-spectra were calculated by dividing the images acquired with off-resonance saturation pulses by the $S_{0}$ image (Eq. 1). 2-D adaptive noise-removal filtering based on [47] was applied to remove the noise of Z-spectra

$Z(\Delta \omega)=\frac{S_{\mathrm{sat}}(\Delta \omega)}{S_{0}}$.

A Lorentzian fitting scheme similar to [48] was applied in our study. The fitting based on a two-pool model (Eq. 2) was performed to define reference Z-spectra by fitting DS and MT effects to the Z-spectra

$$
\begin{aligned}
& Z_{\mathrm{ref}}(\Delta \omega)=1-A_{\text {water }} \frac{L_{\text {water }}^{2} / 4}{L_{\text {water }}^{2} / 4+\left(\Delta \omega-\delta_{\text {water }}\right)^{2}} \\
& -A_{\mathrm{MT}} \frac{L_{\mathrm{MT}}^{2} / 4}{L_{\mathrm{MT}}^{2} / 4+\left(\Delta \omega-\delta_{\mathrm{MT}}\right)^{2}}+b .
\end{aligned}
$$

Here, $A$ is the Lorentzian amplitude, $L$ is the Lorentzian width, $\delta$ is the center frequency, and $b$ is the total shift. Only frequency offsets considered to be affected by the background signal (from DS and MT effects) were used for fitting. For the fit of DS, the images acquired at $\pm 1, \pm 0.5, \pm 0.25$, and $0 \mathrm{ppm}$ were used. For fitting the broad MT effect, the images acquired at $\pm 100, \pm 50, \pm 40$, $\pm 30, \pm 20, \pm 10, \pm 9.5, \pm 9, \pm 8.5$ ppm were used.

LD analysis was used to determine CEST effects: the fitted DS and MT effects were subtracted from the full $\mathrm{Z}$-spectra. The two-pool Lorentzian fit was also used for B0 inhomogeneity correction. In each voxel, LD and Z-spectra were shifted by the frequency shift of the minimum value of the Lorentzian fit. Then, both of them were calculated by interpolating the value from -100 to $100 \mathrm{ppm}$ to a resolution of $0.1 \mathrm{ppm}$ and, for each frequency shift $\Delta \omega$, averaging LD between $\Delta \omega-0.2 \mathrm{ppm}$ and $\Delta \omega+0.2 \mathrm{ppm}$. As a result, $B_{0}$-corrected LD was obtained, from which the APT (3.5 ppm) and NOE (-3.5 ppm) weighted LD maps were generated. After LD analysis, $Z_{\text {ref }}$ and $Z$ were used to 
calculate the $\operatorname{MTR}_{\text {REX }}[34,35,48]$ where APT and NOE signal at +3.5 and $-3.5 \mathrm{ppm}$

$\mathrm{LD}=Z_{\text {ref }}-Z$,

$\mathrm{MTR}_{\mathrm{REX}}=\frac{1}{Z}-\frac{1}{Z_{\mathrm{ref}}}$.

Here, $Z_{\text {ref }}$ was calculated from two-pool fitting as a reference signal, and $Z$ was the normalized $B_{0}$-corrected $Z$-spectra. In the following text, when we talked about APT/NOE imaging under a certain CEST metrics, we refer LD APT/

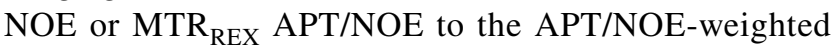
CEST imaging based on LD or MTR $\mathrm{REX}_{\mathrm{R}}$, respectively.

\section{Region of interest analysis}

In the healthy volunteers, whole brain white matter (WM) and grey matter (GM) were used as ROI. In the patients, the contralateral normal appearing WM (NAWM) and GM (NAGM), and tumor were used as ROI. WM/GM tissue segmentation was performed automatically by FAST [49] based on brain extracted $T_{1}$-weighted structural images. The whole brain masks were manually divided into hemispheres. For tumor segmentation in patients, we aligned pre-contrast $T_{1^{-}}$ weighted, $T_{2}$-weighted, and FLAIR images into the space of post-contrast $\mathrm{T}_{1}$-weighted image using the Elastix toolbox (version 2.5) [50]. Based on these four structural images, the tumor masks for non-enhancing glioma were automatically delineated using HD-GLIO [51, 52]. All tumor segmentations were checked by an experienced neuroradiologist. The WM, GM, and tumor ROI were then aligned into CEST space. We computed the mean value of LD APT/NOE and MTR $_{\text {REX }}$ APT/NOE for each ROI in each subject. To evaluate the tissue separation of CEST imaging between ROI (tumor and NAWM/GM), we computed tissue contrast (TC) and contrast-to-noise ratio (CNR) for each patient based on Eqs. 3 and 4 based on LD

$\mathrm{TC}_{\text {tumor,NAWM/GM }}=$ meanLD $_{\text {tumor }}-$ meanLD $_{\mathrm{NAWM} / \mathrm{GM}}$,

$\mathrm{CNR}_{\text {tumor,NAWM/GM }}=\frac{\mathrm{TC}_{\text {tumor,NAWM/GM }}}{\sqrt{\mathrm{std}_{\text {tumor }}^{2}+\mathrm{std}_{\mathrm{NAWM} / \mathrm{GM}}^{2}}}$.

To evaluate the extent of hyper/hypointensity of LD APT/

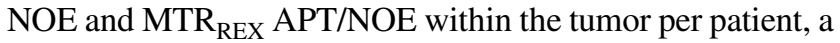
patient-specific threshold was determined as reported in [17]

$\mathrm{LD}_{\text {thresh,hyper }}>\overline{\mathrm{LD}_{\mathrm{APT}, \mathrm{NAWM}}}+2 \times \operatorname{std}_{\mathrm{APT}, \mathrm{NAWM}}$,

$\mathrm{LD}_{\text {thresh,hypo }}<\overline{\mathrm{LD}_{\mathrm{APT}, \mathrm{NAWM}}}-2 \times \operatorname{std}_{\mathrm{APT}, \mathrm{NAWM}}$, where $\overline{\mathrm{LD}_{\text {APT,NAWM }}}$ is the average LD APT in NAWM. The percentage of hyper/hypointense voxels $\left(\mathrm{VP}_{\text {hyper/hypo,CEST }}\right)$ was calculated by dividing the numbers of voxels below $\mathrm{LD}_{\text {thresh,hyper/hypo }}$ within the tumor region by the total number of voxels covering the tumor region which calculated from the structural images for each patient. To assess TC, CNR, and $\mathrm{VP}_{\text {hyper/hypo,CEST }}$ for $\mathrm{MTR}_{\mathrm{REX}}$, Eqs. 5-8 were calculated by substituting $L D$ for MTR $_{\text {REX }}$.

\section{Statistical analysis}

All statistical analyses were performed by SPSS (IBM Corp. Released 2017. IBM SPSS Statistics for Windows, Version 25.0. Armonk, NY: IBM Corp). For comparison of each $B_{1}$ group in the healthy volunteers study, one-way ANOVA was applied to investigate the effects of different $B_{1}$ powers on mean LD APT/NOE in each ROI. Post-hoc analysis was applied to compare the differences of group mean values between $B_{1}$ groups.

In the patient study, mean $\mathrm{TC}$ and mean $\mathrm{CNR}_{\text {tumor,NAWM/NAGM }}$ based on LD APT/NOE and MTR $_{\text {REX }}$ APT/NOE were calculated for all subjects under each $B_{1}$ power. Paired $T$ tests were used to compare mean TC $_{\text {tumor,NAWM/NAGM }}$ and $\mathrm{CNR}_{\text {tumor,NAWM/NAGM }}$ under different $\mathrm{B}_{1}$ powers in patients. One-sample $T$ tests were performed for each TC and CNR. Paired $T$ tests were used to compare $\mathrm{VP}_{\text {hyper/hypo,CEST }}$ under different CEST metrics in patients. One-sample $T$ tests were performed for each $\mathrm{VP}_{\text {hyper/hypo,CEST }}$ for LD APT/NOE and MTR REX $_{\text {APT/NOE. In all statistical }}$ analyses, $p<0.05$ was considered a significant difference.

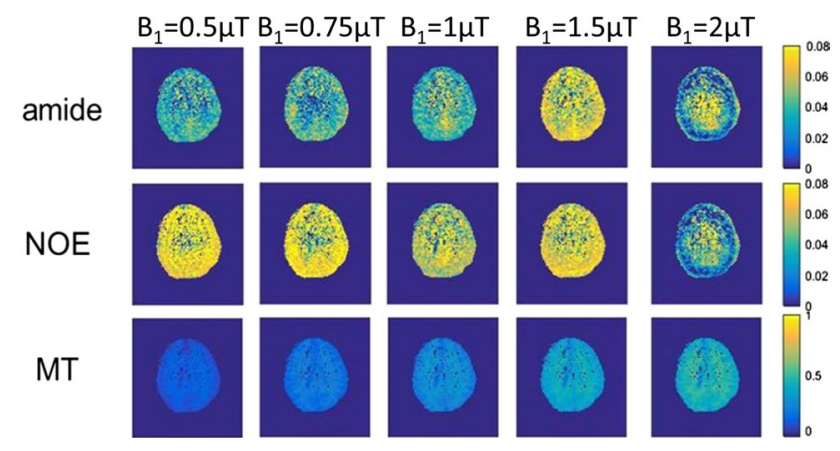

Fig. 1 Example map of different effects, from a single brain slice in a healthy volunteer under different $B_{1}$ powers. Each row corresponding to an effect (from top to bottom: LD APT at $3.5 \mathrm{ppm}$, LD NOE at $-3.5 \mathrm{ppm}$, amplitude of MT pool). Each column corresponds to a $B_{1}$ power 
Fig. 2 Influence of $B_{1}$ power (a, b) and number of RF pulses on $\operatorname{LD}\left(\mathbf{c}, \mathbf{d}\right.$, with $\left.B_{1}=2 \mu \mathrm{T}\right)$ spectrum. The LD was averaged across the WM (a, c) and GM $(\mathbf{b}, \mathbf{c})$ in the brain of a single healthy volunteer
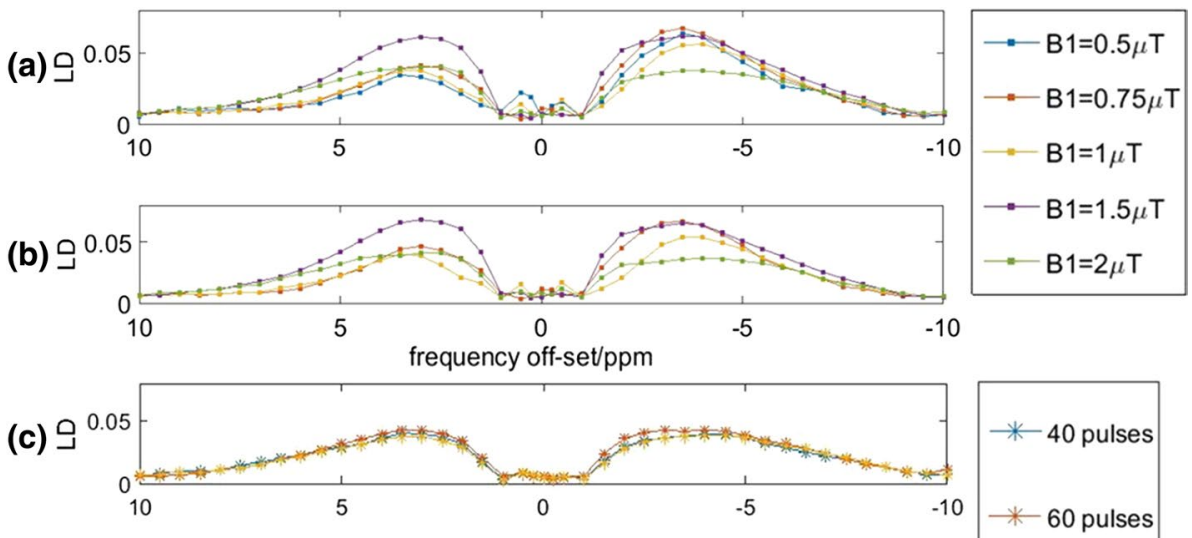

(d)



(a)

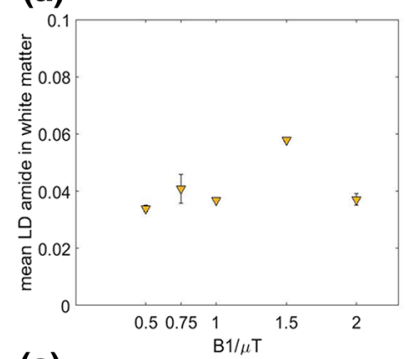

(c)

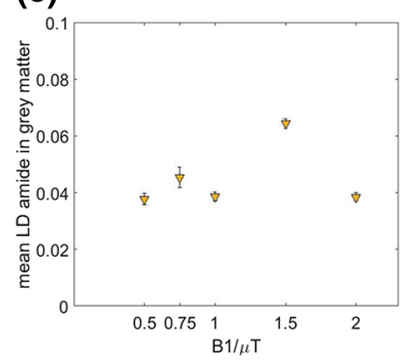

(b)

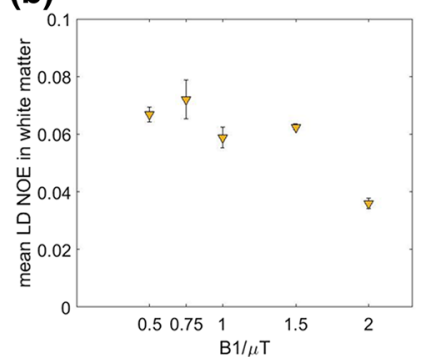

(d)

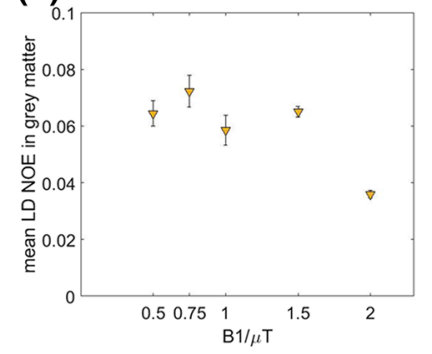

Fig. 3 Group averaged LD APT (a, c) and NOE (b, d) in WM (top row) and GM (bottom row). Error bars represent standard deviation. LD APT was significantly higher $(p<0.05)$ in WM and GM at 1.5 $\mu \mathrm{T}$ compared to any other $\mathrm{B}_{1}$ used. LD NOE was significantly lower $(p<0.05)$ in $\mathrm{WM}$ at $2 \mu \mathrm{T}$ compared to any other $B_{1}$ used

\section{Results}

\section{Healthy volunteers study}

Examples of LD APT, LD NOE, and MT maps acquired with different $B_{1}$ power are shown in Fig. 1 . The mean LD spectra for WM and GM of one volunteer and with different $B_{1}$ powers and RF pulses are shown in Fig. 2. These results

illustrate that LD APT and NOE were both stronger when using $1.5 \mu \mathrm{T}$ compared to using $2 \mu \mathrm{T}$. The number of RF pulses used made only a limited difference to the $Z$-spectra.

Group averaged LD APT/NOE in WM and GM per $\mathrm{B}_{1}$ power used are shown in Fig. 3. The one-way ANOVA showed a significant effect of $B_{1}$ power on LD APT/NOE in WM and GM $(p<0.05)$. In post hoc analysis, it showed that LD APT was significantly higher $(p<0.05)$ in WM and $\mathrm{GM}$ at $1.5 \mu \mathrm{T}$ compared to any other $B_{1}$ used. LD NOE was significantly lower for $2.0 \mu \mathrm{T}$ in WM compared to any other $B_{1}$ power used $(p<0.05)$. The same trend for lowest LD NOE at $2 \mu \mathrm{T}$ was observed in GM, but this finding was not significant.

\section{Patient study}

Examples of resulting images from one patient are shown in Fig. 4. It can be seen that in the tumor region, LD APT is hyperintense under $1.5 \mu \mathrm{T}$, while LD NOE is hypointense under $0.5 \mu \mathrm{T}$. For $\mathrm{MTR}_{\mathrm{REX}}$, APT and NOE hypointensity in the tumor region was found, which was stronger when using $\mathrm{a} \mathrm{B}_{1}$ of $1.5 \mu \mathrm{T}$ compared to $0.5 \mu \mathrm{T}$. The relation between $\mathrm{LD}$ APT/NOE and $\mathrm{B}_{1}$ saturation power was different (Fig. 5). The mean LD spectra of the tumor and NAWM for one patient are plotted in Fig. 5. With increasing $B_{1}$, the increase in LD downfield from on resonance ( $\mathrm{ppm}>0$ ) was larger in the tumor than in NAWM/NAGM, but the LD upfield from on resonance $(\mathrm{ppm}<0)$ showed a stronger decrease in NAWM/NAGM than in the tumor (comparing top and bottom rows in Fig. 5).

To investigate whether tissue contrasts between the tumor and NAWM/NAGM were significant for APT- and NOEweighted imaging separately, one-sample $t$ tests were applied and the results are shown in Figs. 6 and 7. LD APT under 
Fig. 4 Example brain slice of patient 3 (first row: LD APT, LD NOE, MTR REX $_{\text {APT, }}$ MTR $_{\text {REX }}$ NOE, under $0.5 \mu \mathrm{T}$ and $\mathrm{T}_{1}$ pre-contrast image; second row: LD APT, LD NOE, MTR $_{\text {REX }}$ APT, MTR REX NOE under $1.5 \mu \mathrm{T}$ and FLAIR image



Fig. 5 Influence of $B_{1}$ power on mean LD in NAWM $(\mathbf{a}, \mathbf{c})$ or NAGM $(\mathbf{b}, \mathbf{d})$ compared with mean LD in tumor in the brain of a single subject. a, b: $B_{1}=0.5 \mu \mathrm{T}, \mathbf{c}, \mathbf{d}: B_{1}=1.5 \mu \mathrm{T}$
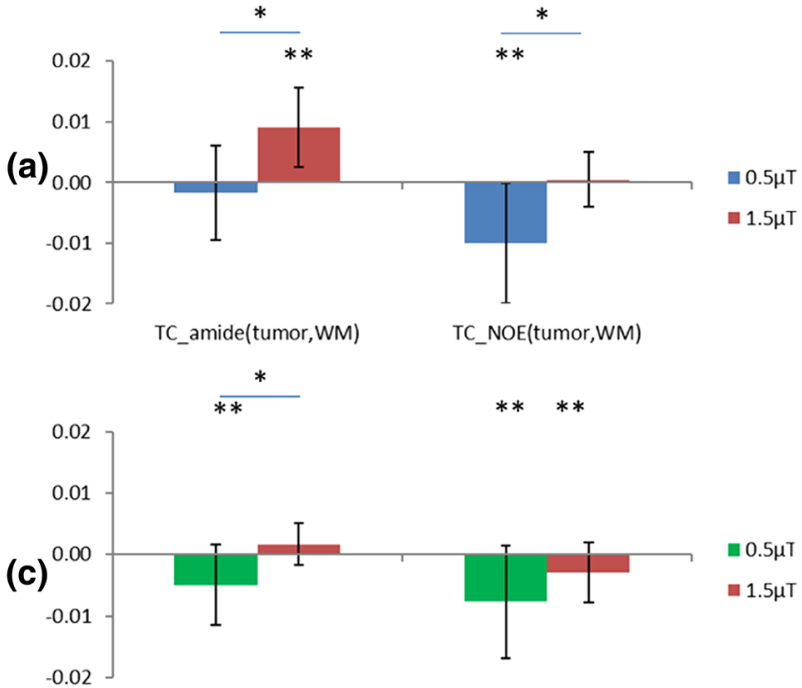

TC_amide(tumor, GM)

TC NOE(tumor,GM)

LD

Fig. 6 Comparison of group averaged $\mathrm{TC}_{\text {tumorNAwM }}(\mathbf{a}, \mathbf{b})$ and $\mathrm{TC}_{\text {tumor,NAGM }}(\mathbf{c}, \mathbf{d})$ for LD APT/NOE (left column) and for MTR $\mathrm{REX}_{\mathrm{R}}$ APT/NOE(right column) each for the two $B_{1}$ powers used (in color).

$1.5 \mu \mathrm{T}$ provided significantly positive $\mathrm{TC}_{\text {tumor,NAwM }}$ and $\mathrm{CNR}_{\text {tumor,NAWM }}$, while under $0.5 \mu \mathrm{T}$, it provided significantly negative $\mathrm{TC}_{\text {tumor,NAGM }}$ and $\mathrm{CNR}_{\text {tumor,NAGM }}$ (Figs. 6 and 7 , left side, $p<0.05, N=10)$. LD NOE under $0.5 \mu \mathrm{T}$ provided significantly negative $\mathrm{TC}_{\text {tumor,NAWM }}$ and $\mathrm{CNR}_{\text {tumor,NAWM }}$, and also significantly negative TC $_{\text {tumor,NAGM }}$ (Figs. 6 a, c and 7a, (b)



(d)

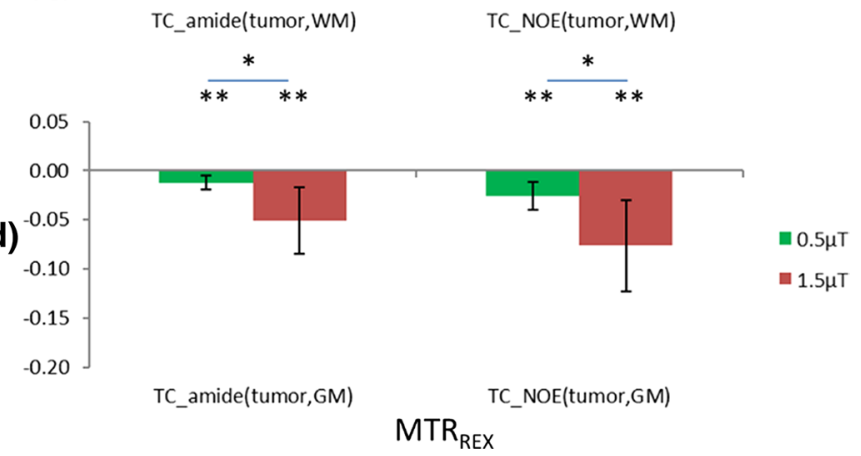

Error bars represent standard deviations. *Significantly different between $B_{1}$ powers, $p<0.05$, **significantly different from $0, p<0.05$

right side, $p<0.05, N=10)$. Under $1.5 \mu \mathrm{T}$, it provided significantly negative $\mathrm{TC}_{\text {tumor,NAGM }}$ (Fig. 6c, right side, $p<0.05$, $N=10)$. $\mathrm{MTR}_{\text {REX }}$ APT/NOE provided significant negative $\mathrm{TC}_{\text {tumor,NAWM/NAGM }}$ and $\mathrm{CNR}_{\text {tumor,NAWM/NAGM }}$ under 0.5 and $1.5 \mu \mathrm{T}$. $\mathrm{MTR}_{\mathrm{REX}}$ showed a stronger increase in both TC and CNR compared with LD (Fig. 6/7, a, c versus b, d). 


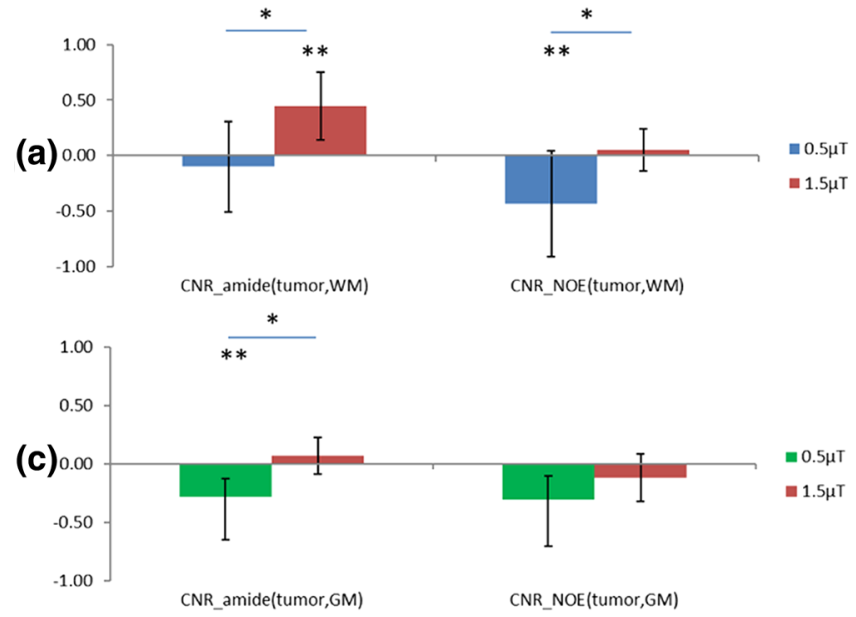

LD

Fig. 7 Comparison of group averaged $\mathrm{CNR}_{\text {tumor.NAWM }}(\mathbf{a}, \mathbf{b})$ and $\mathrm{CNR}_{\text {tumor.NAGM }}$ (c, d) for LD APT/NOE (left column) and for $\mathrm{MTR}_{\mathrm{REX}}$ (right column), each for both $\mathrm{B}_{1}$ powers used. Error bars

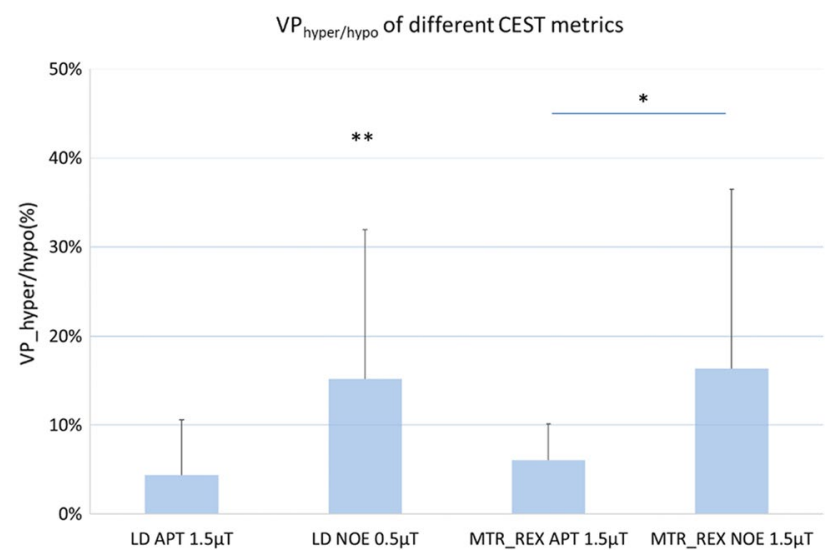

Fig. 8 Group averaged $\mathrm{VP}_{\text {hyper/hypo }}$ for LD APT/NOE and for $\mathrm{MTR}_{\mathrm{REX}}$ APT/NOE. Error bars represent standard deviations. *Significantly different between $\mathrm{MTR}_{\mathrm{REX}} \mathrm{APT}$ and NOE imaging, $p<0.05$, **significantly different from $0, p<0.05$

To compare LD APT/NOE between the two different $B_{1}$ powers, paired $t$ tests were applied and the results can be seen in Figs. 6 and 7. For LD APT, TC tumor,NAwM was positive for $B_{1}=1.5 \mu \mathrm{T}$ and significantly higher than for $0.5 \mu \mathrm{T}$ (Fig. 6a, left side, $p<0.05$, paired $t$ test, $N=10$ ). For LD NOE, $\mathrm{TC}_{\text {tumor,NAWM }}$ was negative for $B_{1}=0.5 \mu \mathrm{T}$ and significantly lower than for $1.5 \mu \mathrm{T}$ (Fig. 6a, right side, $p<0.05$, paired $t$ test, $N=10$ ). In $\mathrm{GM}, \mathrm{TC}_{\text {tumor,NAGM }}$ of LD $\mathrm{NOE}$ in $0.5 \mu \mathrm{T}$ was lower than $1.5 \mu \mathrm{T}$, both are negative. In comparing $\mathrm{CNR}_{\text {tumor,NAWM/NAGM }}$ between the two $\mathrm{B}_{1}$ powers, the same results were found which are shown in Fig. 7. For

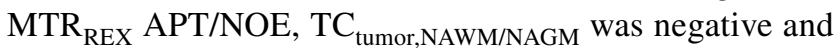
significantly lower at $1.5 \mu \mathrm{T}$ than $0.5 \mu \mathrm{T}$ (Fig. $6 \mathrm{~b}, \mathrm{~d}, p<0.05$,



represent standard deviations. *Significantly different between $B_{1}$ powers, $p<0.05$, **significantly different from $0, p<0.05$

paired $t$ test, $N=10$ ). In comparing $\mathrm{CNR}_{\text {tumor,NAWM/NAGM }}$ of $\mathrm{MTR}_{\mathrm{REX}}$, no significant difference was found between 0.5 and $1.5 \mu \mathrm{T}$.

In the group analysis of $\mathrm{VP}_{\text {hyper/hypo,CEST }}$ (Fig. 8), LD NOE showed significant $\mathrm{VP}_{\text {hypo,CEST }}(15.1 \% \pm 16.7 \%$, tested with one-sample $t$ tests, $p<0.05, N=10)$. MTR $_{\text {REX }}$ NOE showed significant higher $\mathrm{VP}_{\text {hypo,CEST }}$ than MTR $\mathrm{REX}_{\mathrm{RPT}}$ APT $(16.3 \% \pm 23.7 \%$ and $6.0 \% \pm 13.4 \%$, respectively, tested with paired $t$ test, $p<0.05, N=10$ ). No significant difference was found in $\mathrm{VP}_{\text {hypo,CEST }}$ between LD NOE and MTR $\mathrm{REX}_{\mathrm{ROE}}$. No significant difference was found in the contrast of LD APT (with $\mathrm{VP}_{\text {hyper,CEST }}$ of $4.3 \% \pm 6.2 \%$ ) and MTR $\mathrm{REX}_{\text {APT. }}$. No significant difference was found comparing $\mathrm{VP}_{\text {hyper,CEST }}$ resulting from LD APT vs LD NOE.

\section{Discussion}

We found that LD APT with using a $B_{1}$ saturation power of $1.5 \mu \mathrm{T}$ provided significant positive $\mathrm{TC}_{\text {tumor,NAwM }}$ and

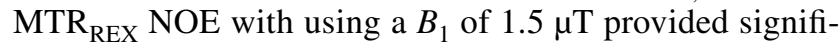
cant negative $\mathrm{TC}_{\text {tumor,NAWM }}$. We also found that $\mathrm{MTR}_{\mathrm{REX}}$ NOE with using a $B_{1}$ of $1.5 \mu \mathrm{T}$ resulted in $16 \%$ of the tumor volume to show hypointense signal, which was significantly

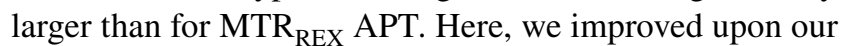
previous work investigating the use of CEST in non-enhancing glioma [17]. The application of a snapshot sequence [43] introduced an even faster acquisition, which allowed us to increase the range of frequency offsets acquired into -100 to $100 \mathrm{ppm}$. This enabled improved MT fitting via the application of a two-pool Lorentzian fitting approach for estimation

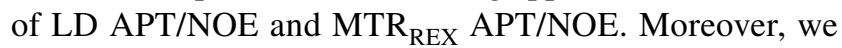


applied lower $\mathrm{B}_{1}$ saturation powers than the one we used previously [17] to increase the weighting of APT and NOE by decreasing the DS and MT effects.

\section{Healthy volunteer study}

Using a $B_{1}$ power of $1.5 \mu \mathrm{T}$ showed strongest contrast for LD APT, both in WM and GM. The reason for the increased trend for LD APT from 0.5 to $1.5 \mu \mathrm{T}$ is likely that higher saturation powers enhance the CEST effect by increasing saturation efficiency $\alpha[53,54]$. However, there is a trade-off for CEST imaging to reach a high APT/NOE effect. Contribution from MT to the saturated CEST signal becomes larger than APT and NOE effects under high $\mathrm{B}_{1}$ power, as suggested in previous simulation work [24, 33]. Our data indicate that this might occur after reaching $B_{1}$ power of 2 $\mu \mathrm{T}$ and that the contribution of MT in WM is approximately ten times as high as APT/NOE effects. This increases the difficulty of isolating APT/NOE signal, hampering accurate fitting of APT/NOE.

For NOE imaging, we found the highest contrast for using $B_{1}$ saturation powers smaller than $2 \mu \mathrm{T}$, with the maximum for $0.5 \mu \mathrm{T}$ which is in line with results from Deshmane et al. [43]. Based on the group averaged LD in Fig. 3, we considered an appropriate $B_{1}$ power for imaging LD APT between 1 and $2 \mu \mathrm{T}$ and a $B_{1}$ of $<1 \mu \mathrm{T}$ for NOE. We selected 0.5 and $1.5 \mu \mathrm{T}$ for the subsequent patient study. We found no differences in LD APT/NOE when reducing the number of RF pulses from 80 to 40 . This can be explained by already having sufficient saturation when using $40 \mathrm{RF}$ pulses in healthy tissue, which helps saving $1.6 \mathrm{~s}$ per CEST image. However, we kept using 80 pulses for glioma imaging in the following patient study to ensure sufficient signal to noise, accepting the slightly longer acquisition time. In addition, in our current set-up we found that using a $B_{1}$ saturation power of 2 $\mu \mathrm{T}$ led to CEST contrast maps that are visibly affected by $\mathrm{B}_{1}$ inhomogeneity. This partly explains why in our set-up using this particular $B_{1}$ power was suboptimal and hence we did not use this saturation power when assessing CEST contrasts in patients.

\section{Patient study}

Our relatively low LD APT values for glioma are in line with what is expected based on previous studies. Note that here we included patients diagnosed with non-enhancing tumors of which nine out of ten were IDH mutated. Finding limited increases in LD APT is therefore in line with previous studies finding isointense or moderate increases in APT-weighted signal in non-enhancing or low-grade glioma $[17,55,56]$. We can expect higher APT-weighted signal in high-grade gliomas [3,55], and thus, better $\mathrm{TC}_{\text {tumor,NAwM }}$ and $\mathrm{CNR}_{\text {tumor,NAWM }}$ will be found in datasets including high-grade gliomas, which often show enhancement on $\mathrm{T}_{1}$-weighted post-contrast images. In addition, care should be taken in comparing the APT-weighted values across different literature. One of the reasons is the definition of $B_{1}$ power. In our study, we used RMS $B_{1}$ across the Gaussianshaped saturation train, similar as used by some other studies $[57,58]$. However, it can also be defined by amplitude of $B_{1}$ pulses used [59] or the mean across the $B_{1}$ pulse train [43]. Another reason for finding discrepancies within the literature is the use of different metrics to evaluate APTweighted signal, such as MTR $_{\text {asym }}$, that are sensitive to other sources than LD APT analysis. This can lead to a different evaluation of APT-weighted signal and different effect sizes to differentiate tumor tissue from NAWM, as investigated previously [17].

In tumor, LD APT showed a stronger increase than LD NOE with increasing B1 from 0.5 to $1.5 \mu \mathrm{T}$. This different sensitivity to $\mathrm{B}_{1}$ illustrates the need of isolating CEST effects for APT and NOE. It also causes a lower TC based on LD NOE at $1.5 \mu \mathrm{T}$ than $0.5 \mu \mathrm{T}$. It is hypothesized that there are more proteins present for proton exchange in the tumor region than in NAWM, which is likely the main contributor for hyperintense APT signal observed in LD APT at $1.5 \mu \mathrm{T}$ in our study $[4,7,60,61]$. A larger increase of LD APT was observed within the tumor region than NAWM when increasing $B_{1}$ power. This can be the reason of the larger LD-based positive $\mathrm{TC}_{\text {tumor,NAwM }}(\sim 1 \%)$ under $1.5 \mu \mathrm{T}$. The saturation time we used was $3.2 \mathrm{~s}$ and sufficient to reach steady state, which can be seen from the LD value near 0 at far-off frequency offsets, achieving the condition for allowing $\mathrm{MTR}_{\mathrm{REX}}$ analysis [36]. MTR $\mathrm{REX}_{\mathrm{APT}}$ showed higher TC and CNR than LD, both under 0.5 and $1.5 \mu \mathrm{T}$, which is most likely due to the correction of spillover effects in $\mathrm{MTR}_{\mathrm{REX}}$ analysis. Interestingly, we found hypointense $\mathrm{MTR}_{\mathrm{REX}}$ APT in the tumor region with using a $B_{1}$ of $1.5 \mu \mathrm{T}$, while for LD APT with the same $B_{1}$ power, we found hyperintense regions within the tumor. This is in line with previous research at $7 \mathrm{~T}$, where LD APT provided significant positive $\mathrm{TC}_{\text {tumor,NAWM }}$ and was decreased with spillover correction [30]. Moreover, the authors explained that APT and NOE imaging in patients with newly diagnose glioblastoma might be showing opposite behavior in tumor contrast because of increased small mobile proteins and decreased large proteins [13]. In our study on non-enhancing glioma, this hypothesis may be reflected by positive $\mathrm{TC}_{\text {tumor,NAWM/NAGM }}$ of LD APT and negative $\mathrm{TC}_{\text {tumor,NAGM }}$ of LD NOE at $1.5 \mu \mathrm{T}$, but not by our $\mathrm{MTR}_{\mathrm{REX}}$ results. Future validation of $L D$ and $\mathrm{MTR}_{\mathrm{REX}}$ in non-enhancing glioma, for instance by increasing the sample size, is needed to investigate whether our current findings will hold.

The LD NOE map showed increases in NAWM and $\mathrm{NAGM}$ at $0.5 \mu \mathrm{T}$ compared to $1.5 \mu \mathrm{T}$. A previous study 
including a low-power saturation experiment $\left(B_{1}=0.6 \mu \mathrm{T}\right)$ at $3 \mathrm{~T}$ showed that NOE signal is significantly lower in the core of a brain tumor compared to contralateral white matter [43]. With using a $B_{1}$ power of $1.5 \mu \mathrm{T}$, we found hypointensity in the tumor area and larger negative $\mathrm{TC}_{\text {tumor,NAWM }}$ for $\mathrm{MTR}_{\mathrm{REX}}$ NOE than for LD NOE, which is again likely reflecting a stronger correction for the spillover effect by $\mathrm{MTR}_{\mathrm{REX}}$ analysis. Such high $M T R_{\mathrm{REX}}$-based $\mathrm{TC}_{\text {tumor,NAWM/NAGM }}$ is in agreement with previous work using MTR $_{\text {REX }}$ NOE at $3 \mathrm{~T}[13,36]$. The origin of hypointense NOE signal can come from low concentration of lipids [30] or the presence of semi-solid proteins with lower mobility in the tumor region. Another origin of such decreased NOE signal can be unfolding of proteins in necrotic regions of the tumor [62]. However, increased numbers of misfolded proteins are often seen in cells with high proliferation rate found in gadolinium enhancing areas, which are of course not present in our nonenhancing glioma data set.

Despite the larger effect size of tissue separation by MTR $_{\text {REX }}$, similar $\mathrm{VP}_{\text {hyper/hypo,CEST }}$ was found when comparing between LD APT and MTR $_{\text {REX }}$ APT at $1.5 \mu \mathrm{T}$. This suggests that LD APT analysis still maintains the ability to detect the possible most aggressive region of tumor, despite fully correcting for the spillover effect. Note that the hyperand hypointense regions resulting from both $\mathrm{MTR}_{\mathrm{REX}}$ and LD confirm the ability of CEST imaging to assess intratumoral heterogeneity of non-enhancing glioma at $3 \mathrm{~T}$. Compared with APT-weighted imaging, higher $\mathrm{VP}_{\text {hypo,CEST }}$ from NOE-weighted imaging may arise from mixed contributions from lipids, low-mobile proteins, and protein unfolding, which can provide additional information for the prediction of physiological environment of the tumor region. With the application of MTR $_{\text {REX }}$, one CEST scan could be sufficient to include both APT and NOE imaging with a clinical feasible time ( $<5 \mathrm{~min})$. The clinical potential of assessing CEST metrics across a tumor volume will lie in assessing which area in the tumor is more aggressive/reflective of the most aggressive part, which can aid taking a biopsy for most accurate diagnosis. In future, this may even aid in identifying tumor regions that already have microscopic tumor invasion, beyond the tumor ROI currently identified solely on structural scans. Further research is needed confirming validation of APT/NOE CEST with targeted biopsies that are not only analyzed by histopathological markers of cell density and proliferation, but also for the underlying protein content with proteomics in human glioma. In combination with reproducibility measurements and prospective, multicenter trials for CEST-based biomarkers such research will further stimulate clinical applications of CEST-MRI, including the use of APT/NOE-weighted CEST for diagnosis and assessment and prediction of therapy response.
A limitation of this study is the small sample size of ten patients, which inhibits investigations of using LD/


glioma. Additionally, we used two-pool Lorentzian fitting and $\mathrm{MTR}_{\mathrm{REX}}$ to evaluate APT/NOE CEST effects. There are other advanced CEST metrics, for example apparent exchange-dependent relaxation (AREX), that compensate for changes in $T_{1}$ relaxation times. However, application of such metrics needs to include $T_{1}$ mapping in the scan protocol, which requires additional sequences and longer acquisition. Suggested by Goerke et al. 36, with the prior knowledge about large spectral range of CEST effects ( $> \pm 6 \mathrm{ppm})$, one can perform Lorentzian fitting at $3 \mathrm{~T}$ without additional advanced fitting approaches such as multistep fitting or MT fitting with special line shapes [43, 63-65]. Finally, here, we did not apply a multi-pool fitting approach ( $>2$ pools) due to the mixed and broadened CEST effects at $3 \mathrm{~T}$. Thus, our LD/MTR $\mathrm{REX}_{\mathrm{R}}$ signal at $3.5 \mathrm{ppm}$ will include other CEST signal sources in addition to amide protons, such as the exchangeable protons from amine, guanidinium, and hydroxyl bonds.

In summary, we applied a CEST pipeline including fast acquisition with snapshot CEST, and individual isolation of APT and NOE effect based on Lorentzian fitting and

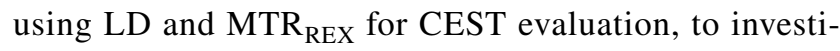
gate the clinical value of CEST imaging on non-enhancing glioma. Appropriate TC and CNR to separate tumor from healthy tissue was found for LD APT and MTR $_{\text {REX }}$ NOE with a $B_{1}$ of $1.5 \mu \mathrm{T}$. We showed that within one CEST acquisition at $1.5 \mu \mathrm{T}(<5 \mathrm{~min})$, the analysis of both $\mathrm{LD}$ APT and MTR $_{\text {REX }}$ NOE allows for observing tumor heterogeneity and provided additional knowledge to conventional structural scans, which will be beneficial in future studies focused on non-enhancing glioma. It is also important to test the reproducibility of CEST imaging for robust diagnosis, e.g., comparing CEST metrics between different patients scanned on different scanners and for longitudinal observations in follow-up of individual patients, which will be included in future work to bring our current pipeline towards clinical application for glioma imaging at $3 \mathrm{~T}$.

Author contributions YW: study conception and design, acquisition of data, analysis and interpretation of data, drafting of manuscript, and critical revision. TCW: study conception and design, analysis and interpretation of data, and critical revision. FA: study conception and design, acquisition of data, and analysis of data. GJB, JAHT: study conception and design, and critical revision. MS, EAHW: study conception and design, analysis and interpretation of data, and critical revision.

Funding This research was conducted with support from the Dutch Cancer Society (KWF): "Non-invasive phenotypying of molecular brain tumor profiles using novel advanced MR imaging and analysis", EMCR 2015-7859, and from the Brain Tumour Charity; "Making the invisible visible: In vivo mapping of molecular biomarkers in adult 
diffuse glioma with CEST MRI", GN-000540. EW is funded by a "Veni Vernieuwingsimpuls" from the Dutch Research Council entitled "Food for thought: Oxygen delivery to the brain”, Grant number 91619121.

\section{Declarations}

Conflict of interest The authors declare that they have no conflict of interest.

Ethical approval All procedures performed in studies involving human participants were in accordance with the ethical standards of the institutional and national research committees and with the 1964 Helsinki declaration and its later amendments or comparable ethical standards. This article does not contain any studies with animals performed by any of the authors.

Informed consent Informed consent was obtained from all individual participants included in the study.

Open Access This article is licensed under a Creative Commons Attribution 4.0 International License, which permits use, sharing, adaptation, distribution and reproduction in any medium or format, as long as you give appropriate credit to the original author(s) and the source, provide a link to the Creative Commons licence, and indicate if changes were made. The images or other third party material in this article are included in the article's Creative Commons licence, unless indicated otherwise in a credit line to the material. If material is not included in the article's Creative Commons licence and your intended use is not permitted by statutory regulation or exceeds the permitted use, you will need to obtain permission directly from the copyright holder. To view a copy of this licence, visit http://creativecommons.org/licenses/by/4.0/.

\section{References}

1. van Zijl PCM, Yadav NN (2011) Chemical exchange saturation transfer (CEST): what is in a name and what isn't? Magn Reson Med 65(4):927-948

2. Zhou J et al (2003) Using the amide proton signals of intracellular proteins and peptides to detect $\mathrm{pH}$ effects in MRI. Nat Med 9(8):1085-1090

3. Togao O et al (2014) Amide proton transfer imaging of adult diffuse gliomas: correlation with histopathological grades. Neuro Oncol 16(3):441-448

4. Jiang $\mathrm{S}$ et al (2019) Identifying recurrent malignant glioma after treatment using amide proton transfer-weighted MR imaging: a validation study with image-guided stereotactic biopsy. Clin Cancer Res 25(2):552-561

5. Jiang S et al (2017) Amide proton transfer-weighted magnetic resonance image-guided stereotactic biopsy in patients with newly diagnosed gliomas. Eur J Cancer 83:9-18

6. Salhotra A et al (2008) Amide proton transfer imaging of 9L gliosarcoma and human glioblastoma xenografts. NMR Biomed 21(5):489-497

7. Yan K et al (2015) Assessing amide proton transfer (APT) MRI contrast origins in $9 \mathrm{~L}$ gliosarcoma in the rat brain using proteomic analysis. Mol Imaging Biol 17(4):479-487

8. Mehrabian $\mathrm{H}$ et al (2018) Evaluation of glioblastoma response to therapy with chemical exchange saturation transfer. Int J Radiat Oncol Biol Phys 101(3):713-723

9. Regnery $\mathrm{S}$ et al (2018) Chemical exchange saturation transfer MRI serves as predictor of early progression in glioblastoma patients. Oncotarget 9(47):28772-28783
10. Meissner J-E et al (2019) Early response assessment of glioma patients to definitive chemoradiotherapy using chemical exchange saturation transfer imaging at 7 T. J Magn Reson Imaging 50(4):1268-1277

11. Paech D et al (2018) Assessing the predictability of IDH mutation and MGMT methylation status in glioma patients using relaxation-compensated multipool CEST MRI at 7.0 T. Neuro Oncol 20(12):1661-1671

12. Paech D et al (2019) Relaxation-compensated amide proton transfer (APT) MRI signal intensity is associated with survival and progression in high-grade glioma patients. Eur Radiol 29(9):4957-4967

13. Goerke $\mathrm{S}$ et al (2021) Clinical routine acquisition protocol for 3D relaxation-compensated APT and rNOE CEST-MRI of the human brain at 3T. Magn Reson Med 86(1):393-404

14. Park KJ et al (2016) Added value of amide proton transfer imaging to conventional and perfusion MR imaging for evaluating the treatment response of newly diagnosed glioblastoma. Eur Radiol 26(12):4390-4403

15. Nakajo $\mathrm{M}$ et al (2021) Correlation between amide proton transfer-related signal intensity and diffusion and perfusion magnetic resonance imaging parameters in high-grade glioma. Sci Rep 11(1): 11223

16. Aum DJ et al (2014) Molecular and cellular heterogeneity: the hallmark of glioblastoma. Neurosurg Focus FOC 37(6):E11

17. Warnert EAH et al (2021) Mapping tumour heterogeneity with pulsed 3D CEST MRI in non-enhancing glioma at 3 T. MAGMA. https://doi.org/10.1007/s10334-021-00911-6

18. Louis DN et al (2021) The 2021 WHO classification of tumors of the central nervous system: a summary. Neuro Oncol 23(8):1231-1251

19. Zhou J et al (2013) APT-weighted and NOE-weighted image contrasts in glioma with different RF saturation powers based on magnetization transfer ratio asymmetry analyses. Magn Reson Med 70(2):320-327

20. Zhao X et al (2011) Saturation power dependence of amide proton transfer image contrasts in human brain tumors and strokes at 3 T. Magn Reson Med 66(4):1033-1041

21. Durmo F et al (2021) Assessment of Amide proton transfer weighted (APTw) MRI for pre-surgical prediction of final diagnosis in gliomas. PLoS ONE 15(12):e0244003

22. Zhou J (2011) Amide proton transfer imaging of the human brain. In: Modo M, Bulte JWM (eds) Magnetic resonance neuroimaging: methods and protocols. Humana Press, Totowa, pp 227-237

23. Zhou J et al (2003) Amide proton transfer (APT) contrast for imaging of brain tumors. Magn Reson Med 50(6):1120-1126

24. van Zijl PCM et al (2018) Magnetization transfer contrast and chemical exchange saturation transfer MRI. Features and analysis of the field-dependent saturation spectrum. Neuroimage 168:222-241

25. Hua J et al (2007) Quantitative description of the asymmetry in magnetization transfer effects around the water resonance in the human brain. Magn Reson Med 58(4):786-793

26. Pekar J et al (1996) Perfusion imaging with compensation for asymmetric magnetization transfer effects. Magn Reson Med 35(1):70-79

27. Swanson SD et al (2017) Molecular, dynamic, and structural origin of inhomogeneous magnetization transfer in lipid membranes. Magn Reson Med 77(3):1318-1328

28. Zaiß M, Schmitt B, Bachert P (2011) Quantitative separation of CEST effect from magnetization transfer and spillover effects by Lorentzian-line-fit analysis of z-spectra. J Magn Reson 211(2):149-155

29. Jones CK et al (2013) Nuclear Overhauser enhancement (NOE) imaging in the human brain at 7T. Neuroimage 77:114-124 
30. Zaiss M et al (2015) Relaxation-compensated CEST-MRI of the human brain at 7T: Unbiased insight into NOE and amide signal changes in human glioblastoma. Neuroimage 112:180-188

31. Zaiss M, Schmitt B, Bachert P (2011) Quantitative separation of CEST effect from magnetization transfer and spillover effects by Lorentzian-line-fit analysis of z-spectra. J Magn Reson 211(2):149-155

32. Desmond KL, Moosvi F, Stanisz GJ (2014) Mapping of amide, amine, and aliphatic peaks in the CEST spectra of murine xenografts at 7 T. Magn Reson Med 71(5):1841-1853

33. Jones $\mathrm{CK}$ et al (2012) In vivo three-dimensional whole-brain pulsed steady-state chemical exchange saturation transfer at $7 \mathrm{~T}$. Magn Reson Med 67(6):1579-1589

34. Zaiss M, Bachert P (2013) Exchange-dependent relaxation in the rotating frame for slow and intermediate exchange-modeling off-resonant spin-lock and chemical exchange saturation transfer. NMR Biomed 26(5):507-518

35. Zaiss M, Bachert $P$ (2013) Chemical exchange saturation transfer (CEST) and MRZ-spectroscopyin vivo: a review of theoretical approaches and methods. Phys Med Biol 58(22):R221-R269

36. Goerke $\mathrm{S}$ et al (2019) Relaxation-compensated APT and rNOE CEST-MRI of human brain tumors at 3 T. Magn Reson Med 82(2):622-632

37. Sun PZ, Sorensen AG (2008) Imaging pH using the chemical exchange saturation transfer (CEST) MRI: correction of concomitant RF irradiation effects to quantify CEST MRI for chemical exchange rate and $\mathrm{pH}$. Magn Reson Med 60(2):390-397

38. Sun PZ, van Zijl PCM, Zhou J (2005) Optimization of the irradiation power in chemical exchange dependent saturation transfer experiments. J Magn Reson 175(2):193-200

39. Heo H-Y et al (2016) Whole-brain amide proton transfer (APT) and nuclear overhauser enhancement (NOE) imaging in glioma patients using low-power steady-state pulsed chemical exchange saturation transfer (CEST) imaging at 7T. J Magn Reson Imaging 44(1):41-50

40. Paech D et al (2014) Nuclear overhauser enhancement mediated chemical exchange saturation transfer imaging at 7 Tesla in glioblastoma patients. PLoS ONE 9(8):e104181

41. Paech D et al (2015) Nuclear Overhauser Enhancement imaging of glioblastoma at 7 Tesla: region specific correlation with apparent diffusion coefficient and histology. PLoS ONE 10(3):e0121220

42. Zaiss M, Ehses P, Scheffler K (2018) Snapshot-CEST: optimizing spiral-centric-reordered gradient echo acquisition for fast and robust 3D CEST MRI at 9.4 T. NMR Biomed 31(4):e3879

43. Deshmane A et al (2019) 3D gradient echo snapshot CEST MRI with low power saturation for human studies at 3T. Magn Reson Med 81(4):2412-2423

44. van der Voort SR et al (2019) Predicting the $1 \mathrm{p} / 19 \mathrm{q}$ codeletion status of presumed low-grade glioma with an externally validated machine learning algorithm. Clin Cancer Res 25(24):7455-7462

45. Jenkinson $\mathrm{M}$ et al (2002) Improved optimization for the robust and accurate linear registration and motion correction of brain images. Neuroimage 17(2):825-841

46. Woolrich MW et al (2009) Bayesian analysis of neuroimaging data in FSL. Neuroimage 45(1 Suppl):S173-S186

47. Lim JS (1990) Two-dimensional signal and image processing. Prentice Hall, Englewood Cliffs

48. Windschuh $\mathbf{J}$ et al (2015) Correction of B1-inhomogeneities for relaxation-compensated CEST imaging at 7 T. NMR Biomed 28(5):529-537

49. Zhang Y, Brady M, Smith S (2001) Segmentation of brain MR images through a hidden Markov random field model and the expectation-maximization algorithm. IEEE Trans Med Imaging 20(1):45-57

50. Klein $\mathrm{S}$ et al (2010) elastix: a toolbox for intensity-based medical image registration. IEEE Trans Med Imaging 29(1):196-205
51. Kickingereder P et al (2019) Automated quantitative tumour response assessment of MRI in neuro-oncology with artificial neural networks: a multicentre, retrospective study. Lancet Oncol 20(5):728-740

52. Isensee $\mathrm{F}$ et al (2021) nnU-Net: a self-configuring method for deep learning-based biomedical image segmentation. Nat Methods 18(2):203-211

53. McMahon MT et al (2006) Quantifying exchange rates in chemical exchange saturation transfer agents using the saturation time and saturation power dependencies of the magnetization transfer effect on the magnetic resonance imaging signal (QUEST and QUESP): Ph calibration for poly-L-lysine and a starburst dendrimer. Magn Reson Med 55(4):836-847

54. Zhou J et al (2004) Quantitative description of proton exchange processes between water and endogenous and exogenous agents for WEX, CEST, and APT experiments. Magn Reson Med 51(5):945-952

55. Choi YS et al (2017) Amide proton transfer imaging to discriminate between low- and high-grade gliomas: added value to apparent diffusion coefficient and relative cerebral blood volume. Eur Radiol 27(8):3181-3189

56. Zou $\mathrm{T}$ et al (2018) Differentiating the histologic grades of gliomas preoperatively using amide proton transfer-weighted (APTW) and intravoxel incoherent motion MRI. NMR Biomed 31(1):e3850

57. Singh A et al (2013) On B1 inhomogeneity correction of in vivo human brain glutamate chemical exchange saturation transfer contrast at 7T. Magn Reson Med 69(3):818-824

58. Kanazawa $Y$ et al (2018) B(1) power optimization for chemical exchange saturation transfer imaging: a phantom study using egg white for amide proton transfer imaging applications in the human brain. Magn Reson Med Sci 17(1):86-94

59. Harris RJ et al (2016) Simulation, phantom validation, and clinical evaluation of fast $\mathrm{pH}$-weighted molecular imaging using amine chemical exchange saturation transfer echo planar imaging (CEST-EPI) in glioma at 3 T. NMR Biomed 29(11):1563-1576

60. Lee DH et al (2017) Quantitative assessment of the effects of water proton concentration and water $\mathrm{T}(1)$ changes on amide proton transfer (APT) and nuclear overhauser enhancement (NOE) MRI: the origin of the APT imaging signal in brain tumor. Magn Reson Med 77(2):855-863

61. Heo HY et al (2017) Insight into the quantitative metrics of chemical exchange saturation transfer (CEST) imaging. Magn Reson Med 77(5):1853-1865

62. Zaiss $\mathrm{M}$ et al (2013) MR imaging of protein folding in vitro employing Nuclear-Overhauser-mediated saturation transfer. NMR Biomed 26(12):1815-1822

63. Heo H-Y et al (2016) Quantitative assessment of amide proton transfer (APT) and nuclear overhauser enhancement (NOE) imaging with extrapolated semisolid magnetization transfer reference (EMR) signals: II. Comparison of three EMR models and application to human brain glioma at 3 Tesla. Magn Reson Med 75(4):1630-1639

64. Zhang $\mathbf{J}$ et al (2018) Improved differentiation of low-grade and high-grade gliomas and detection of tumor proliferation using APT contrast fitted from Z-spectrum. Mol Imag Biol 20(4):623-631

65. Mehrabian H et al (2018) Glioblastoma (GBM) effects on quantitative MRI of contralateral normal appearing white matter. J Neurooncol 139(1):97-106

Publisher's Note Springer Nature remains neutral with regard to jurisdictional claims in published maps and institutional affiliations. 